
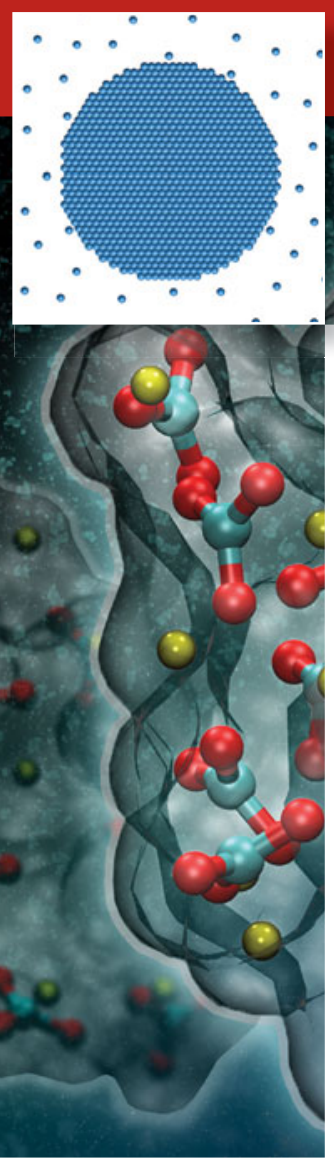

\title{
Nucleation in atomic, molecular, and colloidal systems
}

\author{
Jim De Yoreo and Stephen Whitelam, Guest Editors
}

\begin{abstract}
Nucleation is the first step in the formation of many materials; understanding its microscopic dynamics is crucial for improving synthesis of existing materials and predicting under what conditions novel materials will form. The simple picture of nucleation that prevailed for more than a century does not account for complex nucleation pathways observed in recent years in experiments and simulations. A more general framework is needed to explain reported phenomena; such a framework must account for the peaks and valleys in the free-energy landscape across which nucleation takes place and for the microscopic dynamic factors that dictate how a system explores this landscape. The articles of this issue illustrate and describe the many complex nucleation pathways seen across a range of material systems.
\end{abstract}

\section{The emerging science of materials synthesis}

Research directed toward the predictive science of materials has been largely focused on understanding and manipulating the relationship between structure and function. The goal has been to predict "where the atoms should be placed" in order to achieve a set of properties. In contrast, making materials has generally been pursued through Edisonian approaches, sometimes with the aid of combinatorial techniques. Much less effort has been directed toward the predictive science of materials synthesis, that is, understanding "how to get the atoms where they need to go" in order to achieve a specified structure.

In recent years, the advent of a suite of in situ characterization techniques that can probe synthetic processes at the molecular-to-nanometer scale, ${ }^{1}$ and computational approaches that can simulate processes of cluster formation and particle assembly, ${ }^{2}$ has altered the research landscape, bringing efforts to develop a predictive understanding of synthesis to the forefront. Because nucleation is the seminal event in materials formation, much of the research has focused on this process. ${ }^{3}$

Ordered assemblies of small molecules, macromolecules, or particles that form in solutions from their component parts via the processes of nucleation are materials of major scientific and industrial importance. Such materials include nanowires, nanoparticles and their superlattices, crystalline optical materials and scintillators, porous framework materials, protein crystals, and pharmaceuticals. In order to fabricate better versions of these materials and to predict conditions under which new materials will form, an understanding of the microscopic dynamics of nucleation is crucial.

Although nucleation is a topic whose study dates back to the days of Gibbs, ${ }^{4}$ much of what researchers thought they knew in the last century has been called into question thanks to recent in situ and computational studies. Multistep pathways ${ }^{3}$ involving polynuclear clusters and metastable crystalline, amorphous, or dense liquid states are just some of the phenomena being explored today. These pathways were never envisioned in the classical theory of nucleation.

\section{Adding complexity to the classical theory of nucleation}

Certain basic considerations apply to essentially all molecular systems undergoing nucleation. Moving molecules from the solution into a cluster of molecules (Figure 1a) gives rise to a decrease in free energy that is proportional to the number of molecules in the cluster, scaling with the volume of the cluster. ${ }^{5}$ However, the cluster-liquid interface is, in general, thermodynamically costly, because molecules that sit at the interface possess less entropy than molecules in solution and less favorable energy than molecules in the bulk of the cluster. These factors result in a surface tension or free-energy penalty 

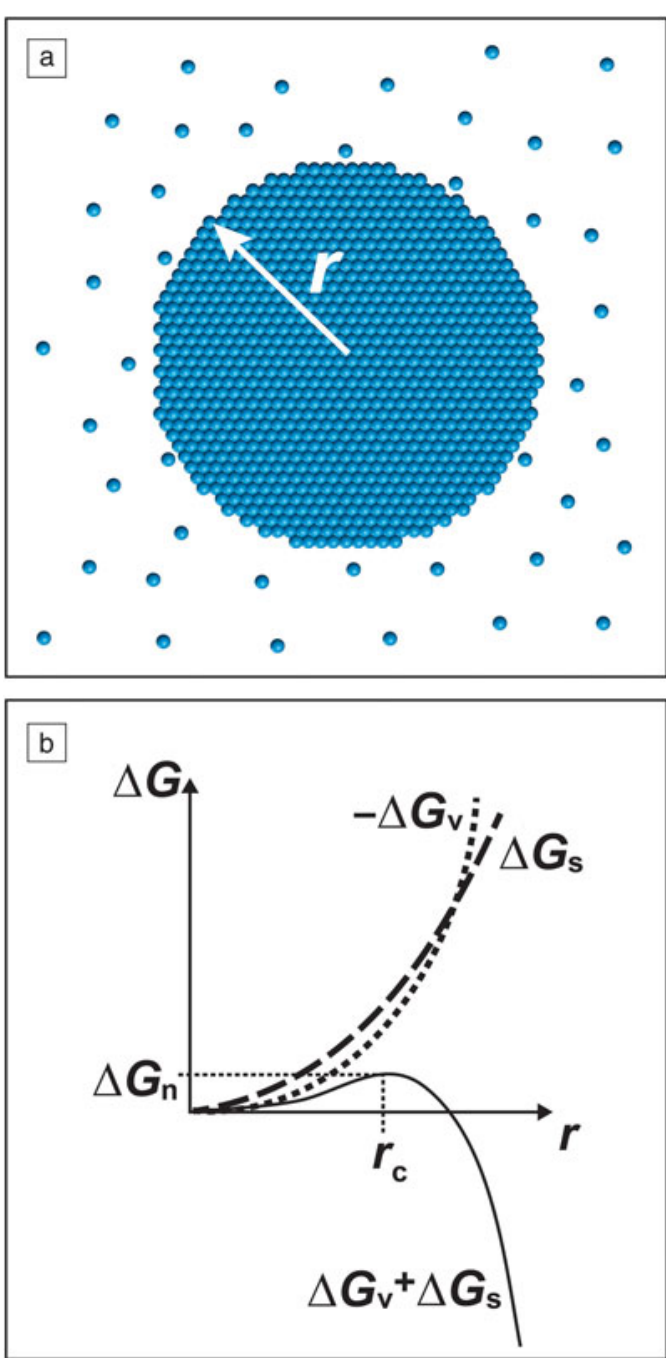

Figure 1. (a) Formation of a circular cluster of radius $r$ from a solution leads to the free-energy changes shown in (b). The crossover of the volume $\left(\Delta G_{v}\right)$ and surface $\left(\Delta G_{\mathrm{s}}\right)$ terms, combined with their opposing signs, leads to a free-energy barrier of height $\Delta G_{\mathrm{n}}$, with a maximum at the critical radius $r_{\mathrm{c}}$ over which the system must pass via thermal fluctuations before the cluster can spontaneously grow.

between cluster and liquid that scales with the surface area of the cluster. The total work of forming the cluster is the sum of these negative and positive terms that are cubic and quadratic in cluster size, respectively (i.e., characteristic linear extent). The quadratic term wins at small size, while the cubic term eventually dominates, leading to a free-energy barrier with a peak at a "critical size," as can be seen in a plot of cluster excess free energy versus cluster size (Figure 1b). The process of crossing this barrier, which must be accomplished by thermal fluctuations of the system, is known as nucleation. ${ }^{6}$

This rather simple picture of nucleation, which dates back to the work of J.W. Gibbs ${ }^{4}$ in the late 1800 s, can be generalized to more complex situations. We can think, instead, of a freeenergy "landscape" for nucleation that depends upon the internal structure of the cluster as well as its size. Thermodynamic factors impose a preference for how a system should proceed across this free-energy landscape, but how it actually crosses this landscape is also determined by dynamic factors (i.e., kinetics), such as how rapidly molecules diffuse and how readily the internal structures of clusters relax. ${ }^{3}$

Understanding nucleation and growth thus entails understanding the thermodynamics and dynamics of molecular association. The interplay of these factors leads to a wide variety of dynamic pathways to assembly (Figure 2). These pathways can be as simple as that envisioned by Gibbs (Figure 2a), or involve, for example, the appearance of thermodynamically metastable particles (Figure 2b) or the emergence of metastable phases (Figure 2c). Which pathway a given system will pursue is certainly tied to the underlying intermolecular and interparticle interactions, but it also depends on external conditions, such as the presence of external surfaces, which can lower the surface tension; the concentration of the solution, which determines the magnitude of the chemical potential that drives nucleation; and the temperature, which controls molecular mobility.

The six articles in this issue of MRS Bulletin illustrate the many complex nucleation pathways seen across a range of materials systems. They capture the state of the field, both in terms of a general understanding of underlying mechanisms, and in the context of distinct materials classes for which structural characteristics dramatically alter the energetic controls from those assumed for simple atomic solids.

\section{Nucleation pathways across diverse materials systems}

In this issue, Sear surveys examples of nucleation in different settings, in both experiments and on the computer, with the goal of elucidating principles that apply across a wide range of materials systems. In experiments, one has little microscopic understanding of how nucleation proceeds in all but the most controlled systems. Nucleation is rare and fleeting, making direct observations challenging, especially because nucleation usually occurs on poorly characterized impurities or surfaces. Faced with these challenges, Sear describes how the application of simple phenomenological models to quantitative experimental data can be used to identify microscopic mechanisms of nucleation. He illustrates how computer simulations can be used to investigate industrially important phenomena, such as polymorphism, in which nucleation results in more than one structure and must be precisely controlled during synthesis of, for example, pharmaceuticals.

Russo and Tanaka, in their article, consider colloidal particles, the model system for which our understanding of nucleation is perhaps most complete. Colloidal particles are large enough and diffuse in liquid slowly enough to be characterized using light microscopy, and their size distribution and interaction range can be controlled to a considerable degree. For these reasons, experiments and simulations of colloidal crystallization provide a testing ground for our understanding 

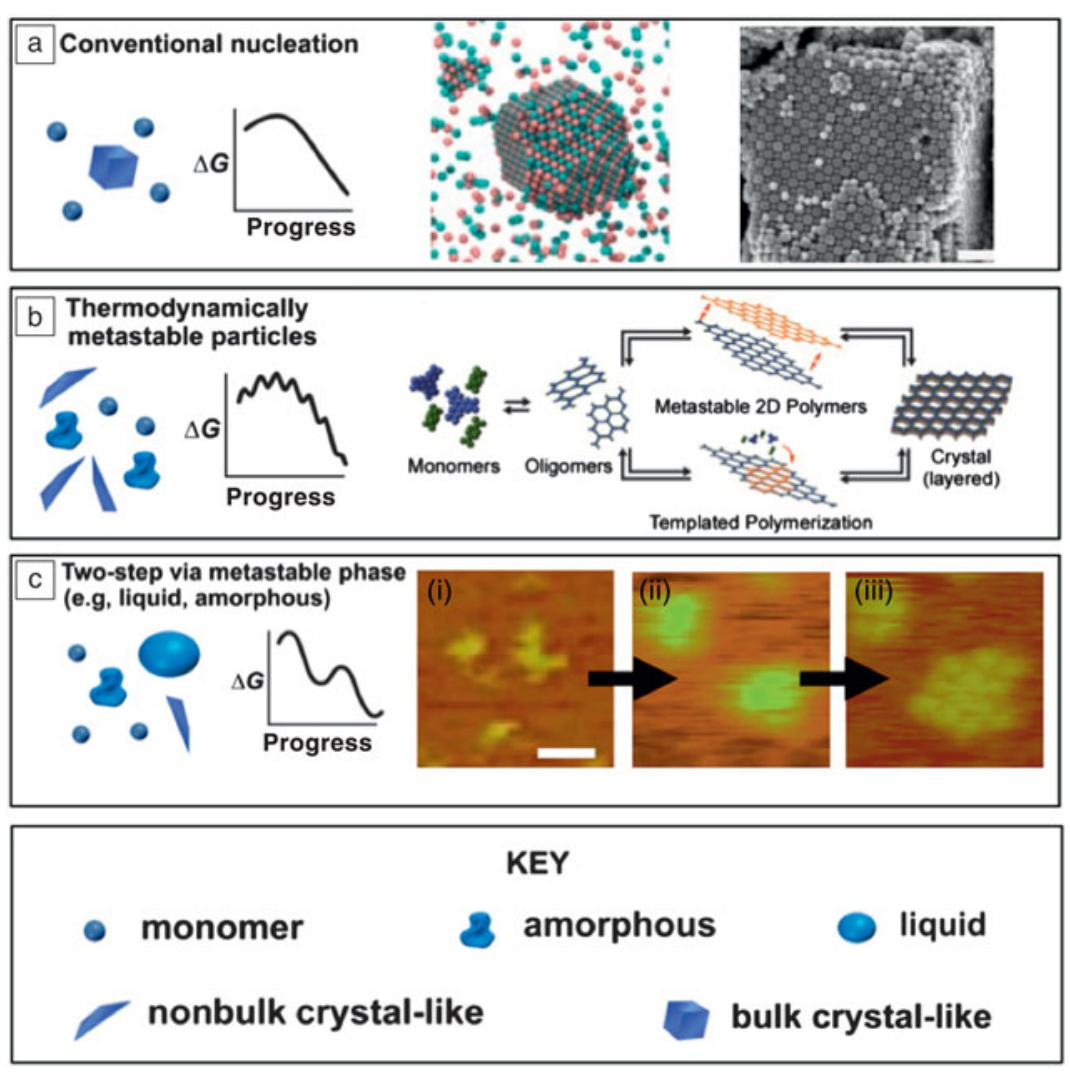

Figure 2. The possible pathways by which monomers form a stable bulk crystal, and the physical mechanisms that give rise to them due to varying types and degrees of complexity in the free-energy landscape. (a) Classical monomer-by-monomer addition with (left) nucleation occurring over a smooth barrier. This is illustrated by (middle) molecular dynamics simulations and (right) a scanning electron microscope image showing formation of a simple colloidal crystal, with a cubic lattice isostructural to $\mathrm{NaCl}$, out of the binary system of DNA-functionalized nanocubes (green) and nanospheres (orange). Reproduced with permission from Reference 7. (c) 2015 Macmillan Publishers Ltd. Reproduced with permission from Reference 8. (c) 2014 Macmillan Publishers Ltd. Scale bar is $200 \mathrm{~nm}$. (b) Aggregation of metastable particles, such as liquid, amorphous, or poorly crystalline particles, or of an oriented (and nearly oriented) attachment of metastable nanocrystals, which occur for (left) nucleation over a barrier with local minima that represent microscopic states of the system higher in free energy than either the solution or final crystal state. This is illustrated by the hypothesized pathway for (right) the covalent organic framework COF-5, for which nucleation occurs through the assembly of monomers and oligomers that then serve as growth units for 2D crystal growth. Reproduced with permission from Reference 9. (C) 2014 American Chemical Society. (c) Crystallization via the formation of a metastable bulk phase, such as a liquid or solid polymorph with (left) two distinct stages of nucleation, the first of which forms a metastable bulk phase before the final crystalline phase nucleates. This is illustrated here by (right) atomic force microscopy images of nucleation of bacterial S-layer membrane proteins into 2D crystals on a lipid bilayer. ${ }^{10}$ The yellow color indicates proteins, which are (i) unfolded, (ii) disordered and liquid-like, and (iii) crystalline. The dark brown is the top of the lipid layer, and the orangeyellow is the S-layer protein diffusing around on the lipids. Scale bar is $25 \mathrm{~nm}$.

of nucleation. Russo and Tanaka survey some of the important behavior seen in colloidal systems, including observations of "two-step" nucleation in which density fluctuations occur before the development of crystalline order. They also describe scenarios in which fluctuations of structural (bondorientational) order occur prior to changes of density. Thus, even in the most controlled model systems, the two order parameters that describe crystallization, density and structure, can evolve in different ways. Therefore, it should not surprise us that increasingly complex behavior is evident as we proceed to systems possessing more structural complexity.

While colloids are essentially rigid, most molecules of industrial or biological interest are conformationally flexible. In their article, Vekilov et al. consider crystallization of such molecules, of which proteins are a prominent example. The authors describe how the processes of conformation change and crystallization can influence each other. For instance, proteins that are able to adopt several conformations when isolated in solution may select a single conformation upon crystallization. This conformation can be unrepresentative of the protein's behavior in solution. For this reason, the structures and properties of proteins in a crystal may be unlike those in solution. Conformation change can also influence the dynamic pathway for self-assembly: Relatively unstructured proteins may associate in a disordered way or as misassembled oligomers, forming dense liquid clusters from which crystals can appear only after proteins adopt "crystallographic" conformations.

Gang and Tkachenko show in their article that DNA offers a synthetic route to dynamic and reconfigurable assemblies. DNA-mediated self-assembly has been used to organize nanoparticles into a wide variety of structures. These include simple crystals with tunable lattice constant and structure, and multicomponent arrays built from anisotropic components. Such control of assembly is made possible by DNA's sequence selectivity. In addition, the ability of DNA to fluctuate and reconfigure in situ allows for the assembly of stimuli-responsive superlattices that are able to change size or shape in response to environmental changes. The authors discuss the impact that simulation and rational design have had on the field of DNAmediated assembly. They anticipate increasingly complex and dynamic superstructures.

In living organisms, nucleation of mineralized tissues generally takes place inside cellular compartments where volumes are constrained and organic surfaces are present. The Whittaker et al. article considers the impact of confinement and organic interfaces on the nucleation process. They illustrate that confinement leads to stabilization of metastable precursor phases, primarily because nucleation rates scale with volume, which is dramatically limited in cellular compartments. However, a number of experiments suggest that stabilization of metastable precursors in confinement must reflect other factors, such as mass-transport limitations. In contrast, experiments with alkane thiol self-assembled 
monolayers and polysaccharide films demonstrate that these surfaces can promote the formation of specific phases, seemingly bypassing metastable phases common in bulk solutions, enhancing rates, and controlling the location of nucleation by reducing interfacial free energies. These insights suggest that living systems may use confined volumes to store metastable amorphous phases and then introduce heterogeneous nucleators to control their transformation.

The value of controlling the size and shape of pores or "negative space" for molecular separation and catalysis is realized in open framework materials, such as zeolites or metal-organic framework compounds, where complex units representing one of many possible end states must assemble from small molecule precursors. In their article, Rimer and Tsapatsis examine the unique challenges associated with nucleation in such systems, highlighting the role of structure-directing agents, particularly in the case of zeolites. The authors discuss how these agents serve as an inverse sacrificial mold around which the framework assembles. In the few zeolite systems for which this assembly process has been deduced, disordered precursors comprise an intermediate phase out of which the ordered units emerge. Construction of the units that comprise the framework from the disordered or amorphous precursors appears to require bond breaking and remaking. Thus, open framework materials highlight the need to introduce chemical reaction networks into theories of nucleation.

\section{Conclusion}

Taken together, these overviews of recent research into the mechanisms and control of nucleation in a range of materials systems highlight the diverse behavior that is manifested.
Classical theories assume that nucleation proceeds through the creation of a small piece of the stable bulk phase. However, many systems exhibit complex assembly pathways that involve transformations between different kinds of material. In some cases, these materials are bulk metastable phases whose probability of formation is as or more likely than that of the most stable phase. In other cases, these materials are nonequilibrium ones that result from microscopic or dynamic factors, such as the conformational flexibility or chemical transformations of a crystal's component parts. Much work remains to be done before these complexities can be rigorously accounted for and the pathways and rates of nucleation of materials predicted. Understanding the full range of this behavior is important for fundamental reasons, but will ultimately benefit a wide range of technologies by enabling the synthesis of new, functional materials.

\section{References}

1. F.M. Ross, Science $\mathbf{3 5 0}$, aaa9886 (2015)

2. F. Giberti, G.A. Tribello, M. Parrinello, J. Chem. Theory Comput. 9, 2526 (2013).

3. J.J. De Yoreo, P.U.P.A. Gilbert, N.A.J.M. Sommerdijk, R.L. Penn, S. Whitelam, D. Joester, H. Zhang, J.D. Rimer, A. Navrotsky, J.F. Banfield, A.F. Wallace, F.M. Michel, F.C. Meldrum, H. Cölfen, P.M. Dove, Science 349, aaa6760 (2015).

4. J.W. Gibbs, A.W. Smith (1874). Trans. of the Connecticut Academy of Arts and Sciences, Volume III, Chapter V, and Chapter IX. New Haven, Tuttle, Morehouse \& Taylor. New Haven: pp. 108-248, pp. 343-524.

5. D. Kashchiev, J. Chem. Phys. 118, 1837 (2003)

6. R.P. Sear, J. Phys. Condens. Matter 19, 033101 (2007).

7. F. Lu, K.G. Yager, Y.G. Zhang, H.L. Xin, O. Gang, Nat. Comm. 6 (2015), doi:10.1038/ncomms7912.

8. E. Auyeung, T.I.N.G. Li, A.J. Senesi, A.L. Schmucker, B.C. Pals, M.O. de la Cruz, C.A. Mirkin, Nature 505, 73 (2014).

9. B.J. Smith, W.R. Dichtel, J. Am. Chem. Soc. 136, 8783 (2014)

10. S. Chung, S.H. Shin, C.R. Bertozzi, J.J. De Yoreo, Proc. Natl. Acad. Sci. U.S.A 107, 16536 (2010).

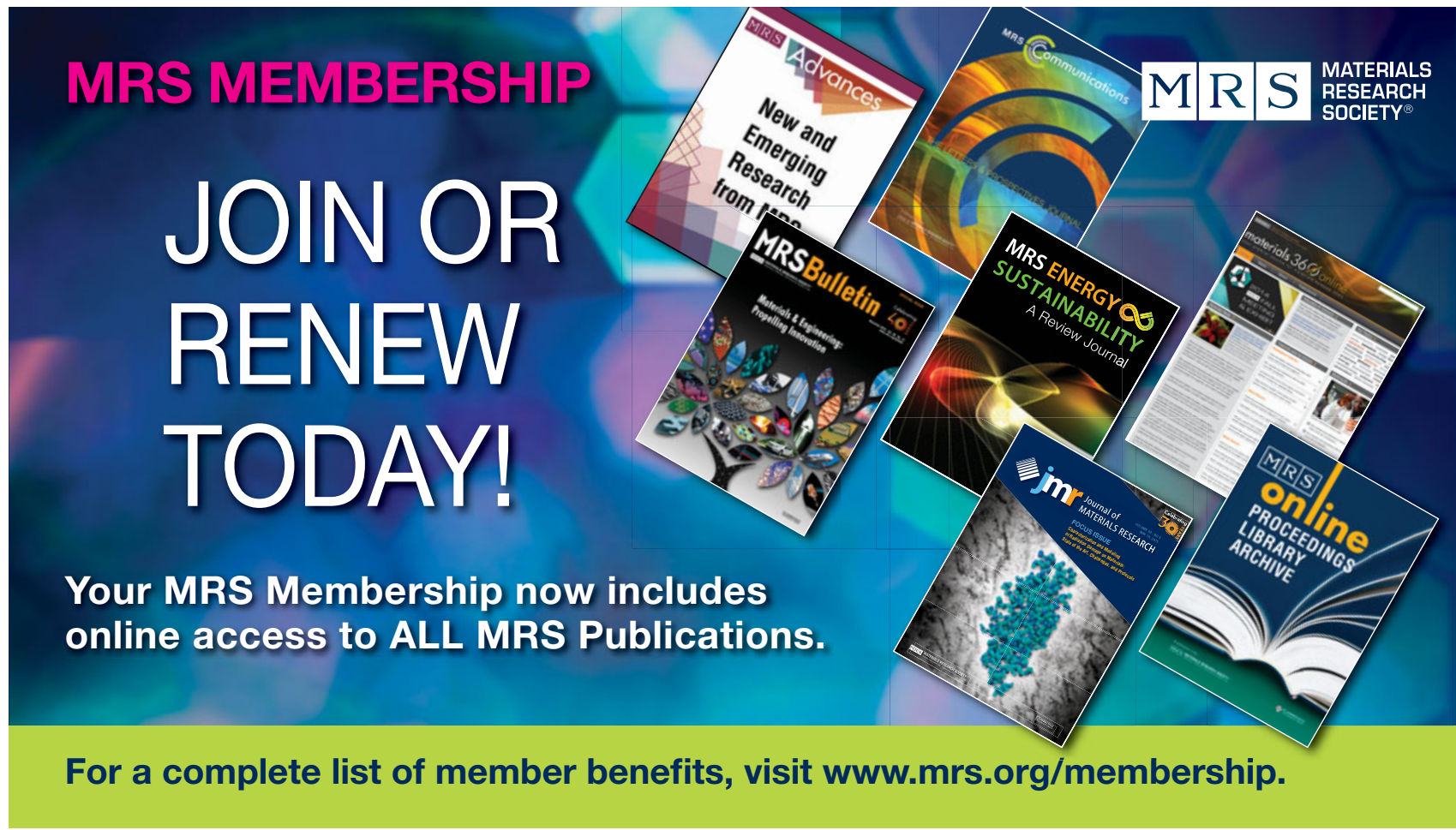

